\title{
Zur Kenntnis des Larieiresinols
}

\author{
von \\ Hugo Hermann.
}

Aus dem Laboratorium für allgemeine Chemie an der $\mathrm{k} . \mathrm{k}$. Technischen Hochschule in Wien.

(Vorgelegt in der Sitzung am 10. Juli 1902.)

Es wurde bereits in einer früheren Abhandlung ${ }^{1}$ mitgetheilt, dass das aus dem Überwallungsharz der Lärche dargestellte Lariciresinol $\mathrm{C}_{17} \mathrm{H}_{16} \mathrm{O}_{4}\left(\mathrm{OCH}_{3}\right)_{2}$ vier freie Hydroxylgruppen enthält, von denen zwei phenolischen, zwei alkoholischen Charakter besitzen. So lässt sich aus genannter Substanz ein Tetraacetylariciresinol $\mathrm{C}_{17} \mathrm{H}_{12}\left(\mathrm{OCH}_{3}\right)_{2}\left(\mathrm{OCH}_{3} \mathrm{CO}\right)_{4}$ gewinnen.

Um einen weiteren Beweis für diese Thatsache $z u$ erbringen, wurde versucht, die im Diäthyllariciresinol

$$
\mathrm{C}_{17} \mathrm{H}_{12}\left(\mathrm{OCH}_{3}\right)_{2}\left(\mathrm{OC}_{2} \mathrm{H}_{5}\right)_{2}(\mathrm{OH})_{2}
$$

vorhandenen zwei freien Hydroxylwasserstoffe durch Acetylgruppen zu ersetzen, wobei zu bemerken kommt, dass genannter Äther in Alkali vollständig unlöslich ist.

Zur Aufhellung der Constitution des Lariciresinols, das in zwei isomeren Modificationen existiert, ${ }^{2}$ war es weiter von Interesse, die Einwirkung alkoholischer Salzsäure auf diesen Körper zu studieren, und habe ich entsprechend dem E.F ischerschen Esterificierungsverfahren ${ }^{3}$ zunächst mit $3 \%$ methyl- und äthylalkoholischer Salzsäure gearbeitet.

$1 \mathrm{~B}$ amberger und Landsied1, Monatshefte für Chemie, XX (1899), 658.

2 Bamberger und Landsiedl, ebenda, XX (1899), 755.

3 E. Fischer, Ber., XXVIII (1895), 3252. 
Bei sechsstündiger Einwirkung erhielt ich aus dem niedrig schmelzenden Lariciresinol in beiden Fällen, aus dem höher schmelzenden Isomeren jedoch nur mit äthylalkoholischer Salzsäure einen inneren Äther des Resinols, das Anhydrolariciresinol. Außer diesem Körper enthielt das Reactionsproduct nur das niedrig schmelzende Isomere, gleichgiltig, von welchem ausgegangen worden war. Auch bei jenem Versuch, welcher keine isolierbaren Mengen des Anhydroproductes lieferte, war nur Lariciresinol vom Schmelzpunkt $104^{\circ}$ nachzuweisen.

Es scheint also, dass der Bildung des Anhydroproductes aus dem höher schmelzenden Isomeren die Umwandlung in das niedrig schmelzende vorangeht.

Das Anhydroproduct wurde methyliert und acetyliert. Es entstanden so ein Dimethyläther und ein Diacetylproduct. Der erstere war nicht acetylierbar, woraus folgt, dass von den ursprünglich vorhandenen vier freien Hydroxylgruppen des Lariciresinols nur zwei übrig geblieben waren, dass also thatsächlich ein innerer Äther vorlag.

Nachdem das Anhydroproduct in Kalilauge löslich ist, war von vorneherein auf den Wasseraustritt aus den alkoholischen Hydroxylgruppen $\mathrm{zu}$ schließen. Diese Folgerung wurde bestätigt durch Überführung des in Kalilauge unlöslichen Lariciresinoldimethyläthers ${ }^{1}$ in Anhydrolariciresinoldimethyläther mittels alkoholischer Salzsäure.

Einer früheren Beobachtung Bamberger's folgend, nach welcher sich das Lariciresinol in rauchender Jodwasserstoffsäure löst, habe ich hierauf die Einwirkung concentrierter wässeriger Chlor- und Jodwasserstoffsäure auf genannten Körper studiert.

Es ergab sich hiebei, dass das höher schmelzende Isomere sich bei weitem leichter löst als das niedrig schmelzende. Aus der Solution erhält man jedoch als einziges Reactionsproduct stets Lariciresinol, Schmelzpunkt 104 bis $108^{\circ}$.

${ }^{1}$ B amberger und L and siedl, Monatshefte für Chemie, XX (1899), 760. 
Die Erscheinungen bei der Lösung machen es wahrscheinlich, dass sich zunächst ein Halogenwasserstoffadditionsproduct bildet, das jedoch beim Versuch, es zu isolieren, sofort in das niedrig schmelzende Isomere übergeht.

Nachdem durch Halogenwasserstoffsäuren der Übergang des einen Isomeren in das andere so leicht bewirkt wird, halte ich für wahrscheinlich, dass das aus beiden, durch Acetylchlorid entstehende Tetraacetylproduct dem niedrig schmelzenden zugehört, da anzunehmen ist, dass der auftretende Chlorwasserstoff das Lariciresinol umlagert.

Anschließend an diese Versuche habe ich die Nitrierung des Lariciresinols studiert und hiebei das bereits bekannte Dinitroguajacol erhaiten ${ }^{1}$ und es erscheint somit im Lariciresinol ebenso wie im Pinoresinol der Guajacolkern nachgewiesen.

\section{Diacetyldiäthyllariciresinol.}

Zur Gewinnung dieses Derivates wurde der Lariciresinoldiäthyläther mit Essigsäureanhydrid längere Zeit gekocht, das Reactionsproduct in Wasser gegossen und der ausgeschiedene feste Körper aus Alkohol umkrystallisiert. Er bildet weiße Nadeln, die bei $113^{\circ}$ schmelzen und in Kalilauge vollständig unlöslich sind. Bei der Verseifung mit alkoholischer Laugewurde der Alkyläther vom Schmelzpunkt $169^{\circ}$ zurückgewonnen.

Die Analyse des bei $90^{\circ}$ getrockneten Acetylproductes ergab nachstehende Resultate:

I. $0 \cdot 3450 g$ Substanz gaben $0.8319 g$ Kohlensäure und $0 \cdot 2179 g$ Wasser.

II. $0.2560 \mathrm{~g}$ Substanz gaben $0.6350 \mathrm{~g}$ Kohlensäure und $0 \cdot 1535 \mathrm{~g}$ Wasser.

1 Herzig, Monatshefte für Chemie, III (1882), 822 und 825 . Bamberger und Landsiedl, Monatshefte für Chemie, XVIII (1897), 488. 
III. $0 \cdot 4130 \mathrm{~g}$ Substanz gaben nach $Z$ eise $10 \cdot 7685 \mathrm{~g}$ Jodsilber. IV. $0 \cdot 2955 \mathrm{~g}$ Substanz gaben nach der Verseifung Essigsäure, welche $8.5 \mathrm{~cm}^{3}$ Natronlauge vom Titer 0.047520 zur Neutralisation brauchte. ${ }^{1}$

In 100 Theilen:

\begin{tabular}{|c|c|c|c|c|c|}
\hline & & Gef & anden & & Berechnet für \\
\hline & I & II & III & IV & $\mathrm{C}_{17} \mathrm{H}_{12}\left(\mathrm{OCH}_{3}\right)_{2}\left(\mathrm{OC}_{2} \mathrm{H}_{5}\right)_{2}\left(\mathrm{OCH}_{3} \mathrm{CO}\right)_{2}$ \\
\hline & $.65 \cdot 80$ & $66 \cdot 74$ & - & - & $66 \cdot 66$ \\
\hline & $.7 \cdot 01$ & $7 \cdot 35$ & - & - & $7 \cdot 00$ \\
\hline $\mathrm{CH}_{3}+\mathrm{C}_{2} \mathrm{H}_{5}$ & - & - & $17 \cdot 42$ & - & $18 \cdot 10$ \\
\hline $\mathrm{C}_{0} \mathrm{H}_{3} \mathrm{O} \ldots$ & - & - & - & $17 \cdot 3$ & $17 \cdot 69$ \\
\hline
\end{tabular}

\section{Methylierung des Lariciresinols mit Dimethylsulfat.}

Lariciresinol wurde in Kalilauge gelöst, mit Dimethylsulfat geschüttelt, und wurden auf diese Weise aus den beiden Isomeren die bereits bekannten Lariciresinoldimethyläther erhalten. ${ }^{2}$

$\mathrm{Zu}$ erwähnen wäre, dass die hiebei aus Lariciresinol, Schmelzpunkt $169^{\circ}$, entstehende butterartige Masse nach monatelangem Stehen erstarrt und dann nach mehrfachem Umkrystallisieren den Schmelzpunkt $167^{\circ}$ zeigt. Es geht also derDimethyläther des höher schmelzenden in den des niedriger schmelzenden Isomeren über.

\section{Einwirkung alkoholischer Salzsäure auf das Lariciresinol.}

Die Vorversuche wurden in gleicher Weise durchgeführt, so dass sie unter einem besprochen werden mögen.

$3 g$ Lariciresinol wurden mit $24 \mathrm{~cm}^{3} 3 \%$ methyl-, beziehungsweise äthyalkoholischer Salzsäure durch 6 Stunden am Rückflusskühler zum Sieden erhitzt.

1 Die Acetylgruppenbestimmung wurde nach der Methode von Wenzel vorgenommen.

2 Bamberger und Landsiedl, Monatshefte für Chemie, XX (1899), 656 und 760 . 
Nach 1 bis 2 Tagen hatte sich eine krystallinische Abscheidung gebildet, die abgesaugt, mit wenig warmen Alkohol ausgelaugt und endlich aus demselben Lösungsmittel umkrystallysiert wurde.

Der so gewonnene Körper schmilzt bei $207^{\circ}$. Er ist in heißem Alkohol ziemlich, in kaltem nur schwer löslich und scheidet sich aus der heiß gesättigten Lösung hauptsächlich am Boden in Form körnig krystallinischer Krusten ab.

Die Analyse der Substanz ergab nachstehende Resultate:

I. $0 \cdot 1874 \mathrm{~g}$ Substanz gaben $0.4798 \mathrm{~g}$ Kohlensäure und $0 \cdot 1064 \mathrm{~g}$ Wasser.

II. $0 \cdot 2023 \mathrm{~g}$ Substanz gaben $0.5156 g$ Kohlensäure und $0 \cdot 1122 g$ Wasser.

III. $0 \cdot 2547 \mathrm{~g}$ Substanz gaben nach Zeisel $0 \cdot 3342 \mathrm{~g}$ Jodsilber.

IV. $0 \cdot 2616 \mathrm{~g}$ Substanz gaben nach Zeisel $0 \cdot 3471 \mathrm{~g}$ Jodsilber.

In 100 Theilen.

\begin{tabular}{|c|c|c|c|c|}
\hline \multicolumn{4}{|c|}{ Gefunden } & \multirow{2}{*}{$\begin{array}{r}\begin{array}{c}\text { Berechnet für } \\
\mathrm{C}_{17} \mathrm{H}_{14} \mathrm{O}_{3}\left(\mathrm{OCH}_{3}\right)_{2}\end{array} \\
\end{array}$} \\
\hline I & II & III & IV & \\
\hline С . ...6.69.79 & $69 \cdot 51$ & - & - & $69 \cdot 51$ \\
\hline H..... $6 \cdot 30$ & $6 \cdot 16$ & - & - & $6 \cdot 09$ \\
\hline $\mathrm{CH}_{3} \ldots \ldots-$ & - & $8 \cdot 38$ & $8 \cdot 48$ & $9 \cdot 15$ \\
\hline
\end{tabular}

Diese Analysenresultate berechtigen, für das in Frage stehende Product die Formel $\mathrm{C}_{17} \mathrm{H}_{14} \mathrm{O}_{3}\left(\mathrm{OCH}_{3}\right)_{2}$ anzunehmen, und stellt dasselbe somit ein Anhydrolariciresinol dar.

Zur Darstellung dieses Körpers verfährt man am besten so, dass man Lariciresinol vom Schmelzpunkt $104^{\circ}$ mit der sechs- bis siebenfachen Menge circa $20 \%$ äthylalkoholischer Salzsäure durch vier Stunden am Rückflusskühler kocht. Nach 24stündigem Stehen wird das ausgeschiedene Anhydrolariciresinol abfiltriert.

Nachdem das so erhaltene Product häufig Spuren einer in Kalilauge unlöslichen Verunreinigung enthält, löst man es 
vortheilhaft in diesem Solvens unter Erwärmen auf, filtriert, fällt mit Salzsäure und krystallisiert aus Alkohol um. Die Ausbeute beträgt $78 \%$ vom Gewicht des angewandten Resinols.

Zur Ermittelung der Zahl der freien Hydroxylgruppen, wurde versucht, ein Acetylproduct und einen Methyläther des Anhydrolariciresinols zu gewinnen.

\section{Diacetylanhydrolariciresinol.}

$2 g$ Anhydrolariciresinol wurden durch 15 Minuten mit $15 \mathrm{~g}$ Essigsäureanhydrid unter Zugabe von etwas Natriumacetat gekocht und hierauf die Reactionsmasse in Wasser gegossen. Der ausgeschiedene Körper wurde aus Alkohol umkrystallisiert.

Das Diacetylanhydrolariciresinol bildet seidenglänzende Nädelchen vom Schmelzpunkt $140^{\circ}$ und ist in Kalilauge unlöslich.

Die Elementaranalyse lieferte folgende Resultate:

I. $0.2677 \mathrm{~g}$ Substanz gaben $0.6565 \mathrm{~g}$ Kohlensäure und $0 \cdot 1399 \mathrm{~g}$ Wasser.

II. $0 \cdot 2516 g$ Substanz gaben $0 \cdot 6155 g$ Kohlensäure und $0 \cdot 1354 \mathrm{~g}$ Wasser.

III. $0.2722 \mathrm{~g}$ Substanz gaben $0.6693 \mathrm{~g}$ Kohlensäure und $0 \cdot 1458 g$ Wasser.

IV. $0 \cdot 2784 \mathrm{~g}$ Substanz gaben nach $Z$ eise $10 \cdot 3020 \mathrm{~g}$ Jodsilber.

V. $0 \cdot 2648 \mathrm{~g}$ Substanz gaben nach Zeisel $0 \cdot 2876 \mathrm{~g}$ Jodsilber.

VI. $0 \cdot 2270 g$ Substanz gaben nach der Verseifung Essigsäure, welche $10 \cdot 6 \mathrm{~cm}^{3}$ Kalilauge vom Titer $0.004157 \mathrm{~g}$ Acetyl zur Neutralisation brauchte.

VII. $0.3872 \mathrm{~g}$ Substanz gaben nach derVerseifung Essigsäure, welche $17 \cdot 68 \mathrm{~cm}^{3}$ Kalilauge vom Titer $0 \cdot 004157 \mathrm{~g}$ Acetyl zur Neutralisation verbrauchte.

VIII. $0.5472 \mathrm{~g}$ Substanz gaben nach der Verseifung Essigsäure, welche $25 \cdot 88 \mathrm{~cm}^{3}$ Kalilauge vom Titer $0.004157 \mathrm{~g}$ Acetyl zur Neutralisation brauchte. 
In 100 Theilen:

\begin{tabular}{|c|c|c|c|c|c|c|c|}
\hline & & & & Inden & & & \\
\hline I & II & III & IV & V & VI & VII & VIII \\
\hline C ........66.98 & $66 \cdot 79$ & $67 \cdot 04$ & - & - & - & - & - \\
\hline $\mathrm{H} \ldots \ldots \ldots .5 \cdot 81$ & $5 \cdot 98$ & $5 \cdot 95$ & - & - & - & - & - \\
\hline $\mathrm{CH}_{3} \ldots \ldots \ldots-$ & - & - & 6.93 & $6 \cdot 93$ & - & - & - \\
\hline $\mathrm{C}_{2} \mathrm{H}_{3} \mathrm{O} \ldots \ldots-$ & - & - & - & - & $19 \cdot 42$ & $19 \cdot 00$ & $19 \cdot 67$ \\
\hline & $\begin{array}{l}\begin{array}{l}\text { Gefun } \\
\text { im } M\end{array} \\
\end{array}$ & ttel & $\mathrm{C}_{1}$ & $\begin{array}{r}\text { Bere } \\
\mathrm{I}_{12} \mathrm{O}(\mathrm{O} \\
\end{array}$ & $\begin{array}{l}\text { hnet fü } \\
\left.\mathrm{H}_{3}\right)_{2} \mathrm{O}\end{array}$ & $\left.{ }_{3} \mathrm{H}_{3} \mathrm{O}\right)_{2}$ & \\
\hline$C \ldots \ldots$ & . $66^{\circ}$ & & & & $8 \cdot 99$ & & \\
\hline$H \ldots \ldots$ & 5 & & & & $5 \cdot 83$ & & \\
\hline $\mathrm{CH}_{3} \ldots \ldots$ & $6 \cdot$ & & & & $7 \cdot 28$ & & \\
\hline $\mathrm{C}_{2} \mathrm{H}_{3} \mathrm{O} \ldots$ & - 19 . & & & & $0 \cdot 87$ & & \\
\hline
\end{tabular}

\section{Dimethylanhydrolariciresinol.}

Anhydrolariciresinol wurde in Kalilauge gelöst und mit Dimethylsulfat geschüttelt. Der ausgeschiedene Äther wurde abgesaugt und mehrmals aus Weingeist und hierauf aus Methylalkohol umkrystallisiert.

Er bildet spießige Krystalle vom Schmelzpunkt $148 \cdot 5^{\circ}$.

Die Analyse lieferte nachstehende Resultate:

I. $0.2360 \mathrm{~g}$ Substanz gaben $0.6045 \mathrm{~g}$ Kohlensäure und $0 \cdot 1465 \mathrm{~g}$ Wasser.

II. $0 \cdot 2205 g$ Substanz gaben $0.5672 g$ Kohlensäure und $0 \cdot 1367 \mathrm{~g}$ Wasser.

III. $0.2094 \mathrm{~g}$ Substanz gaben $0.5376 \mathrm{~g}$ Kohlensäure und $0 \cdot 1298 \mathrm{~g}$ Wasser.

IV. $0 \cdot 3320 \mathrm{~g}$ Substanz gaben nach $Z$ eise $10 \cdot 8145 \mathrm{~g}$ Jodsilber. V. $0 \cdot 2406 \mathrm{~g}$ Substanz gaben nach Zeis e $10 \cdot 6002 \mathrm{~g}$ Jodsilber.

In 100 Theilen:

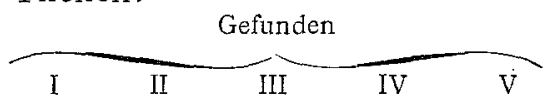

Berechnet für

$\begin{array}{lcccccc} & \text { I } & \text { II } & \text { III } & \text { IV } & \text { V } & \underbrace{\mathrm{C}_{17} \mathrm{H}_{12} \mathrm{O}\left(\mathrm{OCH}_{3}\right)_{4}}_{70.79} \\ \mathrm{C} \ldots \ldots \ldots .69 .86 & 70.15 & 70.02 & - & - & 6.74 \\ \mathrm{H} \ldots \ldots \ldots 6.90 & 6.89 & 6.89 & - & - & 16.85\end{array}$

Denselben Körper erhält man aus Lariciresinoldimethyläther, Schmelzpunkt $167^{\circ}$, durch Kochen mit alkoholischer Salzsäure. 
Einwirkung concentrierter wässeriger Halogenwasserstoffsäuren auf Lariciresinol.

Lariciresinol vom Schmelzpunkt $169^{\circ}$ wurde in kalte rauchende Jodwasserstoffsäure portionenweise eingetragen. Die ersten Partien zerfließen förmlich in der Säure, die späteren lösen sich langsamer auf. Dabei wird die Flüssigkeit dickflüssig und gelatinös. Fährt man mit dem Eintragen fort, so trübt sich die Flüssigkeit und erstarrt endlich vollkommen. Der entstandene Körper ist Lariciresinol vom Schmelzpunkt 104 bis $106^{\circ}$.

Auch das niedrig schmelzende Isomere löst sich in rauchender Jodwasserstoffsäure, obgleich schwieriger und in geringerer Menge.

Beim Eintragen des Resinols in kalte rauchende Salzsäure treten dieselben Erscheinungen zutage.

Ich habe mich vergeblich bemüht, ein etwa intermediär auftretendes Halogenwasserstoffadditionsproduct zu isolieren.

Bei den oben angeführten Versuchen war ein kreosotartiger Geruch zu beobachten.

Ich habe deshalb versucht, durch Einwirkung von concentrierter Salzäure in derWärme eine Spaltung des Lariciresinols zu erzielen, und habe dasselbe (Isomeres, Schmelzpunkt $104^{\circ}$ ) mit concentrierter Salzsäure durch 2, bei anderen Versuchen durch $1 / 2$, beziehungsweise 6 Stunden am Rückflusskühler gekocht und hierauf im Dampfstrom destilliert. Aus dem Destillat konnte durch Extraction mit Äther eine kleine Menge einer intensiv nach Guajacol riechenden Flüssigkeit erhalten werden, die auch mit Eisenchlorid die Farbenreactionen dieses Körpers gab.

Aus dem Destillationsrückstand wurde durch Auskochen mit Wasser unter Zugabe von etwas Alkohol Anhydrolariciresinol isoliert.

\section{Nitrierung des Lariciresinols.}

Concentrierte Salpetersäure verbrennt das Lariciresinol, selbst wenn die Reaction bei $-15^{\circ}$ geführt wird.

Es wurde deshalb dieser Körper unter Kühlung in eine Mischung von drei Theilen Eisessig und ein Theil Salpeter- 
säure (Dichte $=1 \cdot 41)$ eingetragen, bei welcher Operation sich das Resinol anfangs in der Säure ohne merkliche Gasentwickelung auflöst. Später erstarrt die Masse. Nach dem Absaugen krystallisiert man aus Wasser, dem etwas Essigsäure zugesetzt ist, um und erhält so ein in sehr schönen gelben Nadeln krystallisierendes Product vom Schmelzpunkt $122^{\circ}$, welches identisch mit dem zuerst von Herzig dargestellten, später auch von Bamberger und Landsiedl aus Pinoresinol erhaltenen Dinitroguajacol ist.

Die Analyse lieferte folgende Resultate:

I. $0.5000 \mathrm{~g}$ Substanz gaben $0.7219 \mathrm{~g}$ Kohlensäure und $0 \cdot 1361 \mathrm{~g}$ Wasser.

II. $0 \cdot 2014 \mathrm{~g}$ Substanz gaben $24 \mathrm{~cm}^{3}$ Stickstoff bei $19^{\circ}$ und $746 \mathrm{~mm}$.

In 100 Theilen:

\begin{tabular}{|c|c|c|}
\hline \multicolumn{2}{|c|}{ Gefunden } & \multirow{2}{*}{$\begin{array}{r}\text { Berechnet für } \\
\mathrm{C}_{7} \mathrm{H}_{6} \mathrm{O}_{2}\left(\mathrm{NO}_{2}\right)_{2}\end{array}$} \\
\hline I & II & \\
\hline$\ldots \ldots 39 \cdot 38$ & - & $39 \cdot 25$ \\
\hline$\ldots \ldots 3 \cdot 02$ & - & $2 \cdot 80$ \\
\hline$\ldots \ldots-$ & $13 \cdot 41$ & $13 \cdot 09$ \\
\hline
\end{tabular}

Über die im Filtrate vom Nitroproduct enthaltenen Körper wird in einer späteren Abhandlung mitgetheilt werden.

Diese Arbeit hat kurz zusammengefasst nachfolgende Resultate ergeben:

Aus Diäthyllariciresinol lässt sich durch Acetylierung ein Diacetyldiäthyllariciresinol $\mathrm{C}_{17} \mathrm{H}_{12}\left(\mathrm{OCH}_{3}\right)_{2}\left(\mathrm{OC}_{2} \mathrm{H}_{5}\right)_{2}\left(\mathrm{OC}_{2} \mathrm{H}_{3} \mathrm{O}\right)_{2}$ gewinnen.

Lariciresinol (Schmelzpunkt $104^{\circ}$ ) gibt bei der Einwirkung von alkoholischer Salzsäure in der Wärme ein Anhydroproduct, das die Formel $\mathrm{C}_{17} \mathrm{H}_{12} \mathrm{O}\left(\mathrm{OCH}_{3}\right)_{2}(\mathrm{OH})_{2}$ besitzt und von dem das Diacetylproduct $\mathrm{C}_{17} \mathrm{H}_{12} \mathrm{O}\left(\mathrm{OCH}_{3}\right)_{2}\left(\mathrm{OC}_{2} \mathrm{H}_{3} \mathrm{O}\right)_{2}$ und der Dimethyläther $\mathrm{C}_{17} \mathrm{H}_{12} \mathrm{O}\left(\mathrm{OCH}_{3}\right)_{4}$ hergestellt wurden.

Bei der Einwirkung von Halogenwasserstoffsäuren auf Lariciresinol werden sehr leicht zersetzliche Additionsproducte gebildet. 
Lariciresinol gibt mit durch Eisessig verdünnter Salpetersäure ein Nitroproduct, das Dinitroguajacol

$$
\mathrm{C}_{6} \mathrm{H}_{2}(\mathrm{OH})\left(\mathrm{OCH}_{3}\right)\left(\mathrm{NO}_{2}\right)_{2} \text {. }
$$

Herrn Prof. M. Bamberger, sage ich für seine freundliche Unterstützung bei Ausführung dieser Arbeit meinen aufrichtigsten Dank. 